\title{
Impact of Urgent Care Centers on Emergency Department Visits
}

Ramy Yakobi*

Brooklyn Hospital Center, Emergency Medicine, USA

\begin{abstract}
Background: Urgent Care Centers (UCC) and Retail Clinics (RC) have become popular as the need for convenient care continues to grow in light of long waits to be seen by Emergency Departments (ED).

Objective: To analyze the impact of UCC on the ED census of nearby hospitals.

Methods: This retrospective analysis examined census for the EDs located within 2 miles from UCC. The census was obtained for a period of January 2010 through December 2015 from four different hospitals in New York City. This period corresponds to the rapid expansion of UCC in the metropolitan area.
\end{abstract}

Results: Although some variation was seen during certain years, there was an increase in ED census in the year 2015 as compared to that of 2010 . About 100 UCCs operate among the various boroughs of New York City that are capable of addressing illnesses that are classified by ED as ESI Level 4 and 5. Insurance companies would prefer patients to be seen by these centers rather than in costly EDs. Also the overall healthcare cost could potentially benefit if roughly $20 \%$ of the ED visits were seen in UCC. Nonetheless, no impact on total ED census was observed.

Conclusion: While UCCs are relatively new in New York City and the wait time is much shorter for simple illnesses, the presence of UCCs did not decrease the census of main EDs. It is anticipated that these alternative care centers will play a bigger role in the future.

Keywords: Impact; Urgent care centers; Emergency department; Census

\section{Introduction}

A retrospective review of census data was conducted to analyze if Urgent Care Centers (UCC) and other Retail Clinics (RC) in the proximity of hospitals, affected the overall census of the nearby Emergency Departments (ED). This analysis of the impact and the healthcare trends, are a useful tool for ED and hospital administrators to properly allocate resources and adjust staffing.

\section{Background}

Emergency Department (ED) wait times to be seen by a medical provider for patients with non-urgent complaints and turnaround time from registration to final disposition and overall length of visit, are variable and unpredictable [1]. The Center for Medicaid and Medicare Services (CMS) identifies ED length of visit on an average of 160 minutes for "treat and release" patients. Meanwhile, Urgent Care Centers (UCC) offer unscheduled appointments with a "treat and release" length of visits less than 60 minutes [2,3]. As such, the growth of new primary care convenience clinical services such as Urgent Care Center (UCC) and Retail Clinics (RC) is dictated by patients' demand for quality care that is unscheduled, expedited and ubiquitous which makes them accessible and available [4-7]. This created an opportunity for convenience clinics expansion that started in 2010 and approximately 40 additional UCC were planed to open in 2014-2016 in New York City that are operated by healthcare networks and private sector [8,9]. These clinics have developed in all boroughs of metropolitan New York City and provide care for episodic primary care illness. To analyze the effect of UCCs on the total number of ED visits, a retrospective review of census data from hospitals in different boroughs was conducted. The review of data reveals that the ED census did not decline. There are previous articles and research that looked into the travel distance, proximity of freestanding emergency departments as well as the presence of nearby UCCs on the census of the hospitals' EDs. They all demonstrated conflicting conclusions that may not reflect today's trends [10-15].

\section{Methods}

\section{Study design}

A retrospective analysis examined the yearly census of EDs in New York City during the period of 2010 to 2015. This period corresponds to a major expansion of new UCCs as well as RCs located in retail pharmacy chains. While currently in New York City there are over 100 UCCs, not all were operational in 2010, but rather incremental. The popularity and the success of one Urgent Care Center, lead to rapid expansion by same corporations or by other healthcare institutions. To ensure demographic diversity, inclusion criteria were EDs that serve different patient populations. As such, two boroughs which are geographically distant and EDs that range from level I trauma center, to teaching institutions and a community hospital, were sampled. EDs located in similar neighborhoods were excluded. UCC is defined as a healthcare facility that is not EDs, provides primary care to walkin patients without appointments. Most are open daily, i.e., Monday through Sunday, provide onsite $\mathrm{x}$-rays and Point of Care (POC) testing [16]. Most UCCs employ board certified family and/or emergency medicine physicians, physician assistants, medical assistants, $\mathrm{x}$-ray technicians and scribes [17]. RCs are not as equipped as UCCs and are staffed mainly by healthcare advanced practitioners such as Nurse Practitioners and Physician Assistants to attend a simple illness [18]. The location of UCCs selected for this data analysis is in the radius of

*Corresponding author: Ramy Yakobi, Vice Chair, Brooklyn Hospital Center, Emergency Medicine, 120 DeKalb Ave, Brooklyn, N. Y. 11201, United States; Tel: 917886 0839; E-mail: yakobiramy@gmail.com

Received June 04, 2017; Accepted July 27, 2017; Published August 03, 2017

Citation: Yakobi R (2017) Impact of Urgent Care Centers on Emergency Department Visits. Health Care Current Reviews 5: 204. doi: 10.4172/23754273.1000204

Copyright: (c) 2017 Yakobi R. This is an open-access article distributed under the terms of the Creative Commons Attribution License, which permits unrestricted use, distribution, and reproduction in any medium, provided the original author and source are credited. 
1-2 miles from the hospitals. The distance was determined by using Google map. No risks or benefits were attributed to patients as they were not involved. Violation of patient confidentiality did not occur since patient identifiers (name, date of birth, medical record) were not obtained. There was no cost or financial conflict of interest in this census review. As such, no IRB Approval by our Institutional Review Board Approval was required.

\section{Data analysis}

Monthly data of ED census was provided from hospitals registration departments that use Eagle Software Group Inc. The data was aggregated into an annual census and plotted from 2010 to 2015.

\section{Results}

\section{Census analysis from Four EDs}

Data collected from the annual census of four institutions was plotted for the years $2010-2015$. These hospitals are: 1 . Trauma Center Hospital (Level I) 2. Teaching Institution 3. A community hospital 4. Inner city Institution (Figures 1-4). As of early 2015, there were over 100 UCCs and 12 Retail Clinics in New York City (Table 1). Yet, the

ED CENSUS 2010 - 2015

TRAUMA CENTER HOSPITAL

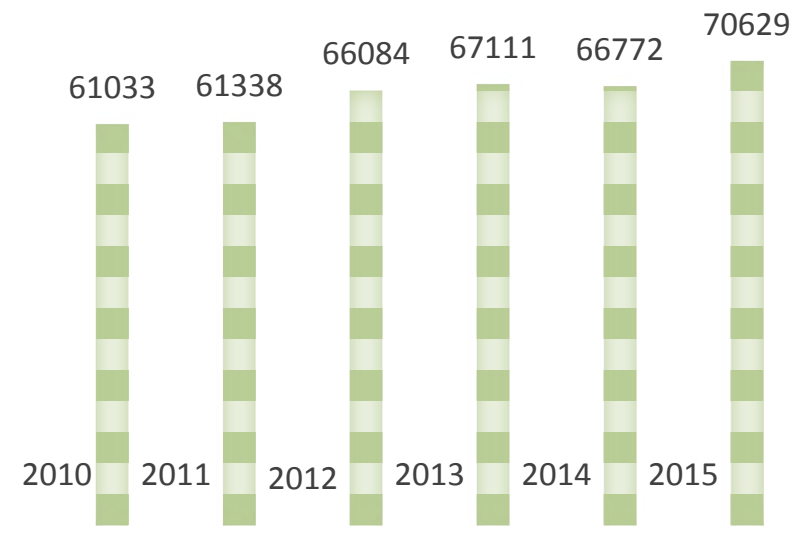

Figure 1: 2010-2015 ED census from Trauma Center Hospital.

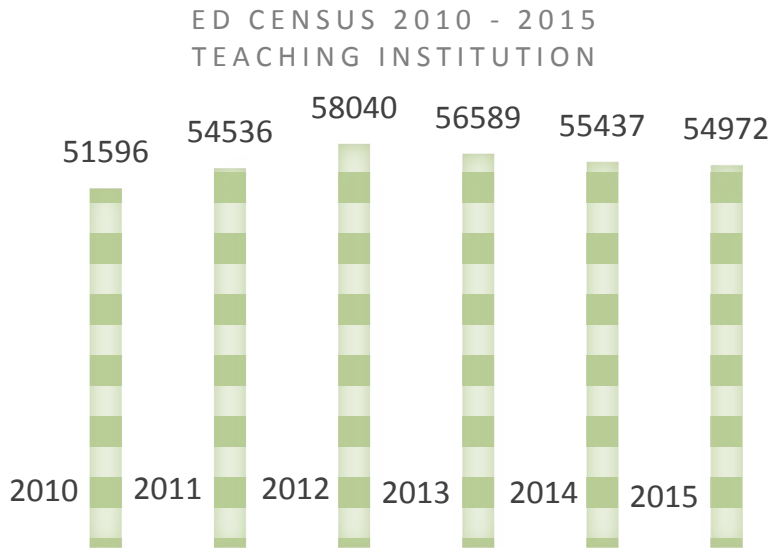

Figure 2: 2010-2015 ED census from Teaching Institution.

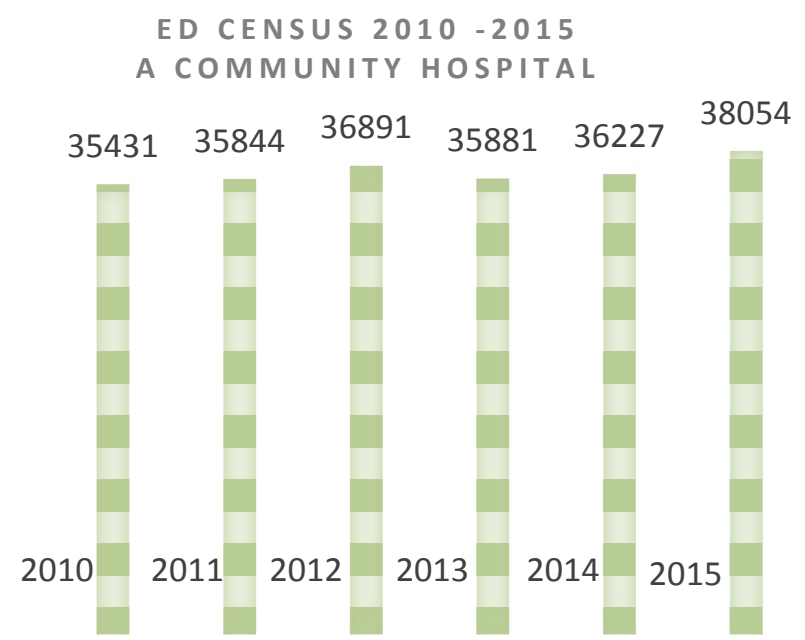

Figure 3: 2010-2015 ED census from a Community Hospital.

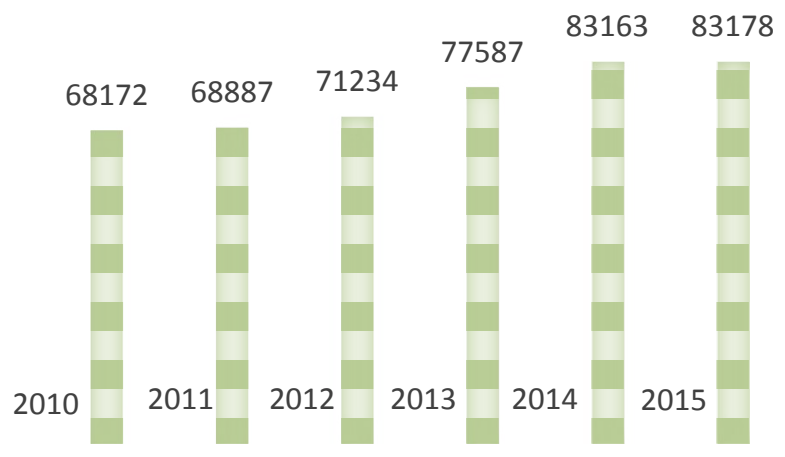

Figure 4: 2010-2015 ED census from Inner City Institution.

\begin{tabular}{|l|c|c|c|}
\hline & New York City & Rest of State & Total \\
\hline Urgent Care Centers & 103 & 263 & 366 \\
\hline Retail Clinics & 12 & 6 & 18 \\
\hline
\end{tabular}

Adopted from United Hospital Fund Convenient Care: Retail Clinics and Urgent Care Centers in New York State Report

Table 1: Convenient care clinics in New York state.

graphs in Figures 1-4 illustrate that while month to month and year to year fluctuation was noticeable in some EDs near the UCCs, the yearly census in 2015 as compared to 2010 was higher. In some EDs the census was progressively incremental over the years during which UCCs were founded and grew in popularity nationwide (Figure 5 and Table 1).

\section{Discussion}

Convenience clinics began to appear 15-20 years ago. Their model was and remains an alternative to consumers who need "on demand" medical attention, however cannot see their PCP due to limited or inconvenient office hours and wants to avoid ED. Convenience clinics operate on the notion that consumers seek and reward simplicity, they want to avoid complexity and unpredictable wait time of the EDs and 


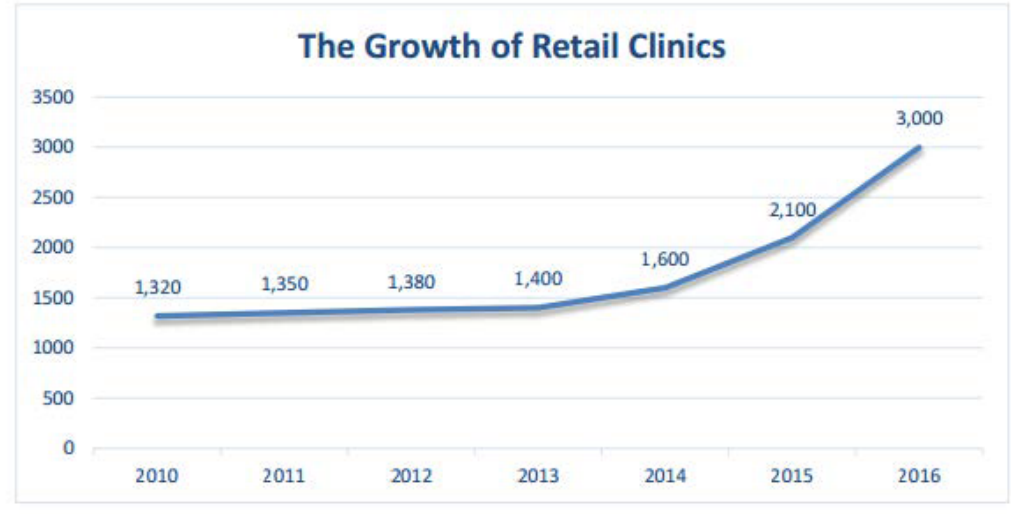

Source: Convenient Care Association

Figure5: Growth of retail clinics.

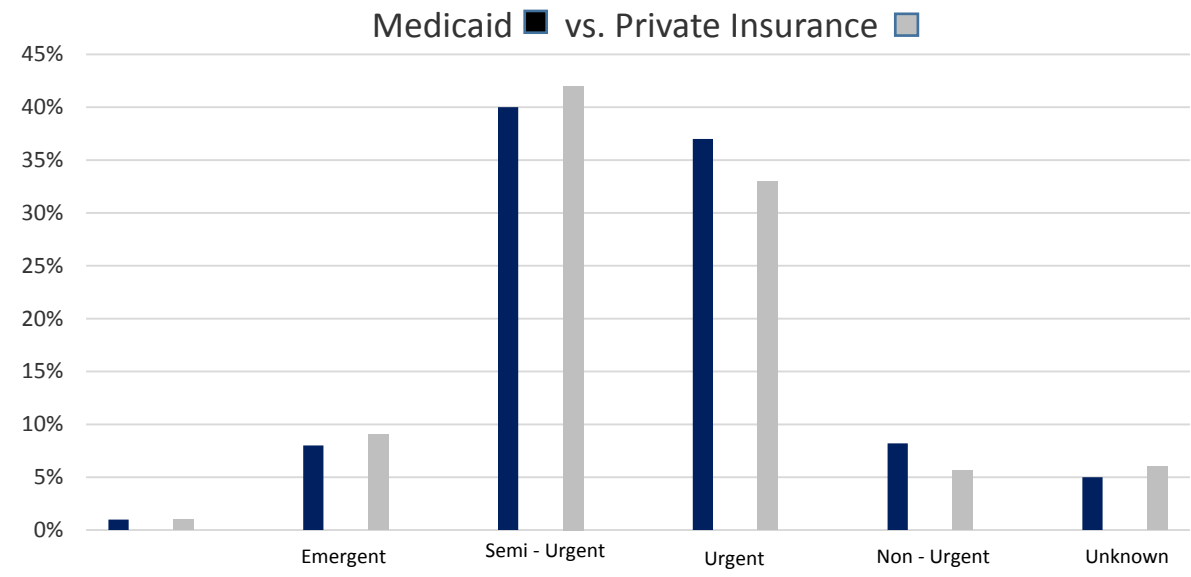

Figure 6: Emergency department visits by triage acuity and insurance carrier (HPA Analysis of 2010, age 0-64).

\begin{tabular}{|l|c|c|c|}
\hline & Retail Clinic & UCC & Primary Care Physician \\
\hline Mehrotta 2009 & $\$ 110$ & $\$ 156$ & $\$ 166$ \\
\hline Thygeson 2008 & $\$ 104$ & $\$ 154$ & $\$ 159$ \\
\hline
\end{tabular}

Source: Adapted from Weinick et al. [29]. Policy implications of the use of retail clinics

Table 2: Costs of care based site.

UCCs offer that convenience [19]. To meet these criteria more than $80 \%$ of convenience clinics are open Monday through Sunday with hours of operation typically from 8 am to $10 \mathrm{pm}$. UCCs offer high-quality care for common episodic illnesses and procedures that range from sprains, flu, colds, ear and throat infections, lacerations, vaccinations and so on [20]. Most of the UCCs provide onsite tests that are considered safe according to Clinical Laboratory Improvement Amendments (CLIA) [21]. The UCC have demonstrated the capability of providing quality care to Emergency Severity Index (ESI) Levels 4 and 5. All this translated to popularity and rapid growth from 2010 through 2016 (Figure 5) [22].

Despite the myth that the EDs are used mainly by uninsured and Medicaid patients, data reveals that the use of EDs by the level of acuity is equal among various payers for same illness, i.e., regardless the insurance, patients seek medical attention for the same complaints [23, 24]. Industry leaders are aware that anywhere between 13.7 to 27.1 (by some estimates even higher) of all ED visits are by private insurance holders and fall into a semi-urgent category (Figure 6). If these patients are shifted away from EDs to UCC, it creates additional growth opportunity for convenience clinics. In turn, this would generate potential cost savings and an alternative to costly hospital ED services (Table 2) [25,26].

As stated earlier, UCCs are open Monday through Sunday and provide onsite $\mathrm{x}$-rays and Point of Care (POC) testing. Most UCCs employees are board certified physicians with a visit length of $60 \mathrm{~min}$. With such a "concierge" model that caters to patient needs and in the process shifts to a lower-cost setting where the quality is as good as the one provided in a traditional setting, one would expect that more patients would seek care in UCC rather than in an ED. Nevertheless, this retrospective census data review conducted to determine whether the census of EDs has been affected by increasing numbers of UCCs in the New York metropolitan area did not reveal any significant impact when compared to a period of 2010 to 2015, a period of major 
expansions of convenience clinics such as UCCs. A literature search revealed few articles that looked into the impact on ED census when UCC or freestanding ED opened in the area. These articles reached a conclusion that ED census has decreased. Most recently however, an ACEP survey revealed that three-quarters of EM physicians report ER visits either flat or going up [27]. Little or no reduction was reported in the volume of low acuity ED visits due to UCCs, retail clinics or even telephone triage [28].

It is difficult to explain the phenomenon why UCCs, telemedicine and RCs do not have an impact on total ED volume. Where to seek medical care depends on symptoms, proximity of healthcare facility, perceived quality, the variety of services available and the type of healthcare insurance. There are forces (regulatory and insurances) trying to keep patients out of the ED and one would expect a shift in ED Census, and yet our data shows no major change, at least not as of yet. It is also unclear whether UCCs complement EDs by referring patients to hospitals. One study revealed that only approximately $3 \%$ of UCC patients were referred to EDs of near by hospitals [29].

\section{Limitation}

The data analysis did not address whether convenience clinics such as UCCs and RCs decreased ED wait time to see a provider nor if the overall length of visit for the same level of urgency, i.e. non-urgent and semi-urgent was affected. It only analyzes if the overall ED census was affected. It is unknown if the payer-mix was affected with patients that are usually seen in the Fast Track section of the EDs (ESI level 5, 4 and some ESI level 3).

\section{Conclusion}

While services offered by UCCs in many ways have similar characteristics to ESI Level 4 and 5 of the EDs, a census data analysis reveals that the presence of UCCs did not lead to a decline in total ED visits to hospitals in their catchment areas. It is unclear from the data provided by the hospitals investigated, if the Levels 4 and 5 visits have decreased. However, as the consumer demands grow, it is unclear what role UCCs will play for patients who can afford them and can be provided with quality care in the UCC settings. As the shortage of physicians will continue to rise (currently Association of American Medical College estimates a shortage of over 20,000 doctors as of 2014), a growing aging population and the average wait time of 18.5 days to see a physician, the need for UCC will grow further [30,31]. In fact, the Research and Development (RAND) Corporation that develops solutions to public policy challenges, estimates that convenience clinics such as UCC that currently offer services to over 80 million U.S. patients, will likely to continue to grow $6 \%$ a year until 2018 [32]. In addition, many hospitals downsize, and as such UCCs may provide an alternative to the use of the ED for certain medical illnesses [33]. What impact this will have on the future ED census, is unknown.

The financial impact of UCCs on healthcare saving is also unknown. We do know that the average cost of an ED visit in 2007 was a low $\$ 500$ vs. the average cost of UCC visit which was $\$ 160$ (Table 2). There are approximately 120 million visits to the U.S. EDs annually and roughly $25 \%$ of those can be treated in UCC. When calculating the monetary difference, the cost savings are estimated to be in billions. Insurance companies are willing to incentivize patients to use UCC rather than Emergency Departments by lowering the co-pay. This too in the future may play a major role on the ED census. Many studies have shown that having medical insurance increases the demand for medical care.
Gaining Medicaid coverage as well as the Affordable Care Act will certainly increase the demand for health care services [32,33]. How this will further affect wait time to the EDs and the PCP, and whether this in turn will shift more patients to the UCCs, remains to be seen.

\section{Article Summary}

- Why is this topic important? - Although it is intuitive to assume that the presence of UCC in the hospital vicinity reduces the census of the ED visits, to date there is no evidence to support this theory. It appears that Hospital and ED leadership do not need to decrease their staffing pattern for now.

- What does this study attempt to show? - Obtaining census data from four EDs in located in demographically distinct neighborhoods, no impact on EDs' census in the vicinity of UCC was observed.

- What are the key findings? - While a rapid expansion of UCC was observed in in last six years, there was no impact on)

- How is patient care impacted? - Although no direct impact on patient care was observed in this study, it is estimated that only $3 \%$ of all the patients seen in UCC are referred to ED. This leads one to conclude that the quality of care in UCC for low acuity illnesses is comparable to ED.

\section{References}

1. Horwitz LI, Green J, Bradley EH (2010) United States emergency department performance on wait time and length of visit. Ann Emerg Med 55: 133-141.

2. Robert M, Goodman MD (2013) Emergency department use associated with primary care office management. AJMC 19: 185-196.

3. Urgent Care Association of America (2013) 25 Things to know about urgent care "2012 Urgent care benchmarking survey results." Urgent Care Industry Information Kit.

4. Weinick RM, Bristol SJ, Des Roches CM (2009) Urgent care centers in the U.S Findings from a national survey. BMC Health Serv Res 9: 79.

5. Weinick RM, Betancourt RM (2007) No appointment needed: The resurgence of urgent care centers in the United States. Oakland, CA: California HealthCare Foundation.

6. Laws M, Scott MK (2008) The emergence of retail-based clinics in the United States: Early observations. Health Aff 27: 1293-1298.

7. Roberts M, Greenblatt $\mathrm{J}$ (2005) Access to urgent medical care among adults 18 years and older, 2000-2002. Agency for Healthcare Research and Quality.

8. Sanchez H (2015) Growing popularity of urgent care clinics has some primary doctors concerned. CBS News.

9. Industry FAQs. Urgent Care Association of America.

10. Brown AM, Decker SL, Selck FW (2015) Emergency department visits and proximity to patients' residences, 2009-2010 NCHS Data Brief, p: 192.

11. Henneman P, Garb J, Capraro G, Li H, Smithline H, et al. (2011) Geography and travel distance impact emergency department visits. J Emerg Med 333339.

12. Berger E (2011) Freestanding emergency departments. Ann Emerg Med 2224A

13. Simon EL, Griffin PL, Jouriles NJ (2012) The impact of two freestanding emergency departments on a tertiary care center. J Emerg Med 43: 1127-1131.

14. Ferber MS, Becker LJ (1983) Impact of freestanding emergency centers on hospital emergency department use. Ann Emerg Med12: 429-433.

15. Merritt B, Naamon E, Morris SA (2000) The influence of an urgent care center on the frequency of ED visits in an urban hospital setting Am J Emerg Med 18 123-125.

16. Weinick RM, Bristol SJ, Marder JE, DesRoches CM (2009) The search for the urgent care center. J Urgent Care Med 38-40. 
Citation: Yakobi R (2017) Impact of Urgent Care Centers on Emergency Department Visits. Health Care Current Reviews 5: 204. doi: 10.4172/23754273.1000204

17. Twenty things-to-Know-About-Urgent-Care-2016.

18. Convenient Care: Growth and staffing trends in urgent care and retail medicine. AMN healthcare report 2015.

19. Chang JE, Brundage SC, Burke GC, Chokshi DA (2015) Convenient care: Retail clinics and urgent care centers in New York state United Hospital Fund.

20. Weinick RM, Bristol SJ, DesRoches CM (2009) Urgent care centers in the U.S. Findings from a national survey. BMC Health Serv Res 9: 79.

21. Information on CLIA Waivers (2008) U.S. Food and drug administration.

22. Understand the Value of an Urgent Care Center-2014.

23. (2015) Review of the evidence on the use of the emergency department by Medicaid patients and the evolving role of emergency medicine physicians prepared for emergency medicine action fund by Health Policy Alternatives, Inc.

24. Sommers AE, Boukus E, Carrier RM (2012) Dispelling myths about emergency department use: Majority of medicaid visits are for urgent or more serious symptoms. Res Brief 23: 1-10.

25. Yee T, Lechner AE, Boukus ER (2013) The surge in urgent care centers: Emergency department alternative or costly convenience. Res Brief 26: 1-6.
26. Strupp D (2008) Study: Huge savings possible by using clinics over ER. Jacksonville Business Journal. New York, NY: American City Business J.

27. ER visits continue to rise since implementation of Affordable Care Act (2015) ACEP. ACA Poll Report.

28. Martsolf G, Fingar KR, Coffey R, Kandrack R, Charland T, et al. (2016) Association between the opening of retail clinics and low-acuity emergency department visits. Ann Emerg Med 69: 397-403.

29. Weinick RM, Burns RM, Mehrotra A (2010) How many emergency department visits could be managed at urgent care centers and retail clinics. Health Aff September 29: 1630-1636.

30. Physician Supply and Demand: Projections to 2020 (2008) Exhibit 33: Growth in primary care supply and demand. Washington, DC: Health Resources and Services Administration.

31. Colwill JM, Cultice JM, Kruse RL (2008) Will generalist physician supply meet demands of an increasing and aging population? Health Aff 27: 232-241.

32. Dine AA, Einav L, Finkelstein A (2013) Health insurance experiment three decades later 27: 197-222.

33. A Matter of Urgency (2010) Reducing emergency department overuse. New England Health Institute Research Brief. 